\title{
Does Depression Cause Obesity or Does Obesity Prompt Depression?
}

\author{
Nevzat DEMİRCi* \\ Faculty of Sports Sciences, Mersin University, Turkey \\ *Corresponding author: nevzatdemirci44@ hotmail.com
}

\begin{abstract}
Both depression and obesity are health problems frequently encountered all around the world. The studies carried out in recent years indicate that there is a relationship between obesity and depression but a complete cause and effect relationship cannot be determined. The purpose of this research is to seek an answer to the question of does depression cause obesity o $r$ does obesity prompt depression. Studies were found using PubMed, PsycINFO, and EMBASE databases and selected on several criteria. When the relationship between depression and obesity is examined, the most basic question is which one affects the other one, in other words what the direction of the relationship between them is. Consequently: studies indicate that there is a relationship between obesity and depression and focus on cause and effect relationship. It is argued that this relationship is bilateral, and it is stated that many factors can affect this relationship. It has been found that obesity increases the depression risk, and depression is also a predictor factor for the development of obesity. In conclusion, it can be said that there is not a single cause for the relationship between obesity and depression, and it has a multi-factorial structure.
\end{abstract}

\section{Keywords}

Obesity, Depression, Health, Body Mass İndex

\section{INTRODUCTION}

Significant progress has been made in improving public health around the world; However, many serious health problems such as depression and obesity need to be solved. The global prevalence of being overweight and obese was estimated to be $39 \%$ and $13 \%$ in 2017 for adults 18 years and older, respectively (Ha et al., 2017). In 2018, the World Health Organization (WHO) estimates that depression affects approximately 300 million people worldwide (Cui et al., 2018). Obesity; Due spent than more calories, characterized by fat tissue growth in the body, which reduces the quality of life by preparing the ground for organic disorder is a public health problem of significant and avoidable (Garaulet et al., 2010) The prevalence of obesity in Turkey was reported to be $32 \%$.

In our country, adults It has been shown that $2 / 3$ of the population is overweight or obese, weight gain is more common in men and obesity is more common in women. In 2016, the World Health Organization (WHO) stated that more than half of the world's population was obese or overweight. reported that more than 42 million children under five years of age suffer from overweight or obesity (Çakmur and Güneş, 2018).

Depression, which is defined as a deeply sad mood disorder that negatively affects the thoughts, feelings and behaviors of the individual, is considered as another public health problem and its incidence is increasing gradually. (WHO, 2019) It has been reported that 350 million people in every age group are affected by depression. The increasing prevalence of depression and obesity is important because both conditions increase the risk of systemic disease (Luppino et al., 2010) The preventable and manageable nature of obesity and depression is the development of policies in primary health care that protect and improve health and thus reduce health expenses (Baughman et al., 
2003) A number of molecular, genetic, hormonal, immunological and environmental factors have been investigated in the etiology of depression and obesity (Gesta et al., 2007) In recent years, a causal relationship between obesity and depression has been investigated.

Many studies have been reported to examine whether or not. (Lang et al., 2015; Onyike et al., 2003). Both depression and obesity are major health issues which require particular attention in order to reduce the global burden. In terms of mechanisms, a growing body of literature sustains that depression and obesity share some epidemiological, clinical and biological pathways (Milaneschi et al., 2018) in a bidirectional manner, with obesity increasing the risk of depression and depression increasing the risk of obesity in prospective studies (Wurtman and Wurtman, 2018). The purpose of this research is to seek an answer to the question of does depression cause obesity o $r$ does obesity prompt depression.

\section{Obesity of Epidemıology}

Obesity is defined as excess body fat. Body mass index (BMI) is the standard measure of obesity and overweight in children 2 years and older. BMI is divided by square length, which is equal to body weight. In adults, a BMI between 25 and 30 is considered overweight, a BMI equal to or greater than 30 is considered obese. In children, BMI varies according to age and gender. Obesity in children is defined as a BMI greater than or equal to 95 percent for age and gender. As children approach adulthood, the percentage of BMI in age and gender is approaching adult standards (Stunkard et al., 2003) Countries across the world have experienced a significant increase in the prevalence of overweight and obese children and adolescents from the 1980s to the 1990s.

Evidence from the United States reveals that this upward trend continues until the 21st century (Janssen et al., 2005). A dramatic increase in the prevalence of obesity among adolescents in the United States 1976 The prevalence of overweight and obesity in 1980 and 2007-2008 (Ogden et al., 2010), 1986 and 1998, Increased $120 \%$ for blacks and Hispanics, 50\% for whites and 50\% for whites. Although obese girls strive to reduce the increasing prevalence during puberty, rates are still increasing and the stigma of obese children is increasing. Currently, almost one-third of children and adolescents in the United States are either overweight or obese. The factors affecting the outbreak of obesity mainly include an increase. changes in diet and food consumption such as inactivity and fast feeding patterns and large portion sizes (Melnyk et al., 2009).

\section{Epidemiology of Depression}

The risk of depression increases in adolescents and the prevalence of major depressive disorder (MDD) is $2 \%$ in children and $4 \%$ to $8 \%$ in adolescents. a rate comparable to the lifetime prevalence of MDD in adults. Young girls are likely to experience more depression than adolescents during adolescence. Gender differences occur in early adolescence and persist in adulthood (Reeves et al., 2008). Criteria for depression include mood, anhedonia, fatigue, feelings of guilt or worthlessness, thoughts of death, sleep and appetite changes, or psychomotor activity.

Problems with sleep, appetite, or psychomotor activity can occur in either direction (ie, a person's insomnia or hypersomnia; anorexia or increased appetite; psychomotor retardation or agitation). The DSM-IV-TR criteria for MDD require that five of the nine depression criteria are often present for a period of 2 weeks; One of the criteria should include either depressive mood or anhedonia (ie, decreased interest or pleasure) and symptoms should be a change from previous functioning (Hankin et al., 1998; APA, 2000)

\section{Obesity-Depression Cycle}

Obesity and depression are therefore common conditions that have significant individual effects. Taken together, it has been shown that depression and obesity affect the quality of life negatively in a synergistic way (Romain et al., 2018). A two-way relationship has been proposed between the two, but not all studies support this, suggesting that the relationship is a complex one (Singh 2014). Increased severity of depression has been shown to be associated with decreased physical activity and increased caloric intake, resulting in an increased risk of obesity.

Obesity can be used to predict a weaker response to antidepressants and a worse depression outcome for 1 year; One particular factor involved is the development of metabolic syndrome (Jantaratnotai 2017). Both depression and obesity are common health problems worldwide. Recent 
studies indicate that there is a relationship between complete cause-effect relationship. When the relationship between obesity and depression is examined, the most important question is which one affects the other, ie the direction in which the relationship is between them. Some studies suggest that there is a corresponding relationship between these two variables; some are depression risk factors for obesity; others found that obesity is a risk factor for depression (Bartlett et al., 2017).

\section{Effect of Obesity and Depression}

For years, any association between obesity and depression has been assumed to be largely random in the general population, but a recent subanalysis by Luppino and colleagues (Faith et al., 2002) found that the impact of obesity on depression development was stronger in American studies. They emphasized the possibility of a biological link between overweight, obesity and depression; obesity was seen as an inflammatory condition. Inflammation has also been associated with depression, which is perceived as a stressful obesity and depression, but cannot identify a living event in which the brain responds similarly to a medical disease, leading to high proinflammatory cytokines.

In normal overweight people, adipose tissue contains adipose cells, but in obese people adipose tissue remains macrophages, pathogens and other impurities, and the immune system is released continuously by releasing inflammatory hormones such as TNF-alpha and interleukin-6. contributes to the situation (Bastard et al., 2006). Luppino and colleagues also noted that the biological mechanisms underlying the risk of onset of obesity and depression may not be different across cultures, but that sociocultural systems may be different and more stringent in one culture compared to other cultures. According to the National Health and Nutrition Examination Questionnaire (NHANES) -III data, among the most obese adolescents, 95-100. In percentages, the prevalence of majordepression has increased to $20 \%$ for boys and $30 \%$ for girls (Stunkard et al., 2003)

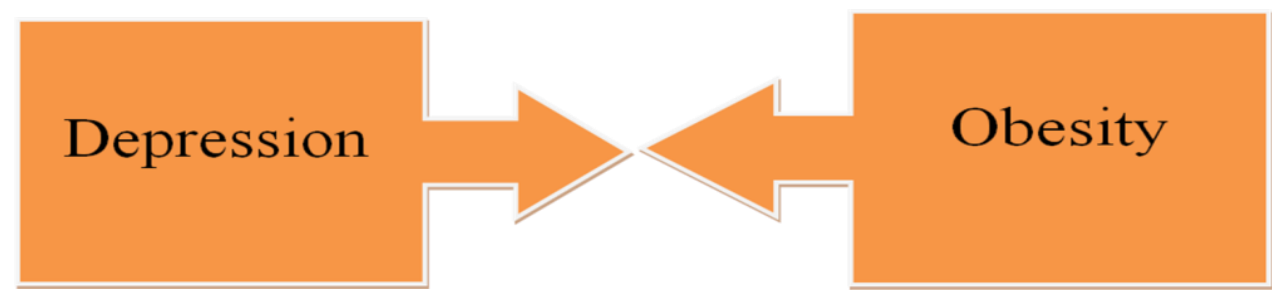

Figure 2. Longitudinal studies support the possibility of two-way causal interaction.

Archives of General Psychiatry - The results of a new metanalysis have shown that obesity and depression are closely linked, and that an increase in one increases the risk for another. In obese people, the risk of developing depression increases by $55 \%$, and in people with depression, the risk of developing obesity increases by 58\% (Stunkard et al., 2003). In a review study examining in which direction the relationship between obesity and depression provides more consistent information, 15 studies indicating that depression causes obesity and 10 studies indicating that obesity causes depression have been examined. As a result of this study, $80 \%$ of the studies showed that obesity causes depression; 53\% reported that depression causes obesity. In the general population, it was concluded that being obese increases the risk of developing depression (Faith et al. 2011). In another longitudinal study involving 9374 adolescents; In the first measurements obtained cross-sectionally, there was no significant relationship between depression and obesity; When the measurements taken one year later, depressive mood predicted obesity (Goodman and Whitaker 2002). Obesity and depression are just one area of concern, and the following statistics indicate the scale of the problem:

- $13 \%$ of the world's population is obese (WHO 2017a)

- Over 300 million people worldwide become depressed (WHO 2017b)

- $23 \%$ of obese individuals have comorbid depression (Carey 2014) 
- The prevalence of obesity is as high as $55 \%$ - As independent risk factors, depression constitutes a relative risk of obesity of $37 \%$ and obesity of $18 \%$; however, the risk of overweight in depression is low (Mannan 2015)

\section{Conclusions}

When the relationship between depression and obesity is examined, the most important question is which one affects the other, ie the direction of the relationship between them. Studies indicate a relationship between obesity and depression, while emphasizing cause-effect relationship. It is suggested that this relationship is among adults with severe mental health problems.

bilateral and many factors may affect this relationship. Obesity increases the risk of depression; depression was also found to be a predictor for the development of obesity. In summary, it can be said that the relationship between obesity and depression is not a single cause, it has a multi-factor structure.
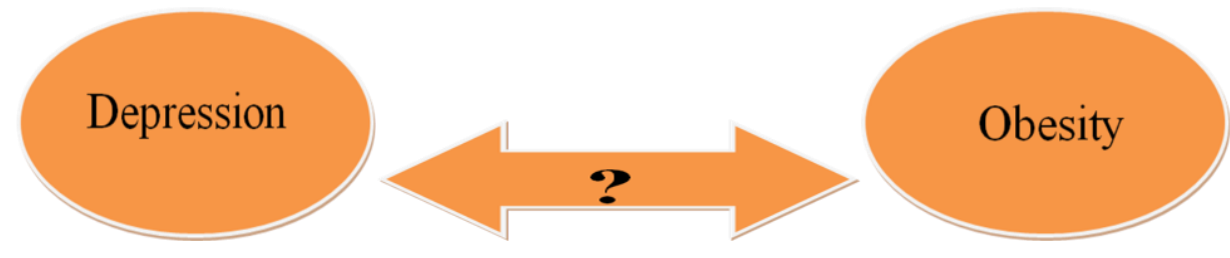

\section{REFERENCES}

American Psychiatric Association (2000). Diagnostic and Statistical Manual of Mental Disorders: DSMIV- TR. 4. Washington, DC.

Bartlett A.A, Singh R, Hunter R.G (2017). Anxiety and Epigenetics. Adv. Exp. Med. Biol., 978, 145-166.

Bastard JP, Maachi M, Lagathu C, Kim MJ, Caron M, Vidal H, Capeau J, Feve B (2006). Recent advances in the relationship between obesity, inflammation, and insulin resistance. Eur Cytokine Network; 17(1):4-12.

Baughman K, Logue E, Sutton K, Capers C, Jarjoura D, Smucker W. (2003). Biopsychosocial characteristics of overweight and obese primary care patients: do psychosocial and behavior factors mediate sociodemographic effects? Prev Med;37(2):129-37.

Carey M, Small H, Lin Yoong S, Boyes A, Bisquera A, Fisher RS (2014) Prevalence of comorbid depression and obesity in general practice: a cross sectional survey. British Journal of General Practice, 64(620): e122-7. Cui J, Sun X, Li X, Ke M, Sun J, Yasmeen N, Khan JM, Xin H, Xue S and Baloch Z (2018) Association Between Different Indicators of Obesity and Depression in Adults in
Qingdao, China: A Cross-Sectional Study. Front. Endocrinol. 9:549.

Çakmur H, Güneş ÜB (2018). Examination of the relationship between obesity and depression in outpatient admissions. Türk Aile Hek Derg; 22 (2): 58-65.

Demirci N, Toptaş Demirci P, Demirci E (2017). The Effect of School-based Exercise Practices of 9-11 Year Old Girls Students on Obesity and Health-related Quality of Life.

Universal Journal of Educational Research 5(8): 1323-1331.

Faith MS, Matz PE, Jorge MA (2002). Obesitydepression associations in the population. $\mathbf{J}$ Psychosom Res; 53:935-942.

Garaulet M, Ordovás JM, Madrid JA (2010). The chronobiology, etiology and pathophysiology of obesity. Int $\mathbf{J}$ Obes ;34:1667-83.

Gesta S, Tseng YH, Kahn CR (2007). Developmental origin of fat: tracking obesity to its source Cell;131:242-56.

Goodman E ve Whitaker RC (2002). A prospective study of the role of depression in the development and persistence of adolescent obesity. Pediatrics 110(3): 497504. 
Ha H, Han C, Kim B (2017). Can Obesity Cause Depression? A Pseudo-panel Analysis. J Prev Med Public Health;50:262-267.

Hankin BL, Abramson LY, Moffitt TE, Silva PA, McGee R, Angell KE (1998). Development of depression from preadolescence to young adulthood: emerging gender differences in a 10-year longitudinal study. J Abnorm Psychol; 107(1):128-140.

Janssen I, Katzmarzyk PT, Boyce WF, Vereecken C, Mulvihill C, Roberts C, Currie C, Pickett W (2005). Comparison of overweight and obesity prevalence in school-aged youth from 34 countries and their relationships with physical activity and dietary patterns. Obes Rev; 6:123-132.

Jantaratnotai N, Mosikanon K, Lee Y, McIntyre RS (2017) The interface of depression and obesity. Obesity Research \& Clinical Practice, 11: 1-10.

Lang UE, Beglinger C, Schweinfurth N, Walter M, Borgwardt S (2015). Nutritional aspects of depression. Cell Physiol Biochem;37(3):1029-43.

Luppino FS, de Wit LM, Bouvy PF, Stijnen T, Zitman FG (2010). Over-weight, obesity, and depression: a systematic review and meta-analysis of longitudinal studies. Arch Gen Psychiatry; 67:220-29.

Mannan M, Mamun A, Doi S, Clavarino A (2015) Is there a bi-directional relationship between depression and obesity among adult men and women? Systematic review and biasadjusted meta analysis. Asian Journal of Psychiatry, 21: 51-66.

Melnyk BM, Jacobson D, Kelly S, O'Haver J, Small L, Mays MZ (2009). Improving the mental health, healthy lifestyle choices, and physical health of Hispanic adolescents: a randomized controlled pilot study. J Sch Health; 79(12):57-584.

Milaneschi Y, Simmons WK, Van Rossum EFC, Penninx BW (2018). Depression and obesity: evidence of shared biological mechanisms. Mol Psychiatry.
Ogden CL, Carroll MD, Curtin LR, Lamb MM, Flegal KM. (2010). Prevalence of high body mass index in US children and adolescents, 2007-2008. JAMA ; 303:242249.

Onyike CU, Crum RM, Lee HB, Eaton WW (2003). Is obesity associated with major depression? Results from the third national health and nutrition examination survey. Am J Epidemiol;158:1139-47.

Reeves GM, Postolache TT, Snitker S (2008). Childhood obesity and depression: connection between these growing problems in growing children. Int $\mathrm{J}$ Child Health Hum Dev.; 1(2):103-114.

Romain K, Webb T, Kumar M (2018). Depression and obesity: can the cycle be broken? BJPsych Advances, vol. 24, 132-140

Singh G, Jackson CA, Dobson A, Mishra GD (2014) Bidirectional association between weight change and depression in mid-aged women: a population- based longitudinal study. International Journal of Obesity, 38: 591-6.

Stunkard AJ, Faith MS, Allison KC (2003). Depression and obesity. Biol Psychiatry; 54:330-337.

Toptaş Demirci P (2018). The Effect of Exercise Behavior Change Processes in the Prevention of Obesity in Elderly. Int $\mathbf{J}$ Disabil Sports Health Sci; 1(2);40-48

World Health Organization (2019). Depression: a global public health concem. http://www.who.int/mental_health/managem ent/depression/who_paper_depression_wfmh 2012.pdf/ adresinden 11/12/2019.

World Health Organization (2017a) Obesity and overweight [Online Fact Sheet]. WHO (http://www.who.int/mediacentre/factsheets/f s311/en/). Accessed May 2017.

World Health Organization (2017b) Depression [Online Fact Sheet]. WHO (http://www.who.int/mediacentre/factsheets/f s369/en/). Accessed May 2017.

Wurtman J, Wurtman R. (2018). The Trajectory from Mood to Obesity. Curr Obes Rep; 7:1-5. 